\title{
“STATUS" DE CONSERVACIÓN DE LAS ESPECIES VEGETALES SILVESTRES DE USO TRADICIONAL EN LA PROVINCIA DE CANTA, LIMA-PERÚ
}

\author{
CONSERVATION "STATUS" OF THE WILD VEGETAL SPECIES OF \\ TRADITIONAL USE IN THE PROVINCE OF CANTA, LIMA-PERU
}

\author{
Horacio De la Cruz Silva ${ }^{1}$, Percy A. Zevallos Pollito ${ }^{2}$ y Graciela Vilcapoma Segovia ${ }^{3}$
}

\begin{abstract}
Resumen
Se determinó el status de conservación de 104 especies de uso tradicional de la provincia de Canta-Lima. El estudio se desarrollo en los años de 2003, 2004 y 2005, siguiendo la metodología del CDC (1991) y UICN (1998 \& 2002) modificadas para las condiciones de la región y del Perú. Las variables tomadas en consideración fueron: distribución geográfica, abundancia, antigüedad de colecciones, localización en áreas expuestas extrativismo, endemismo, confinamiento, presencia en unidades de conservación y protección in situ. Los resultados muestran que 22 especies $(21.2 \%)$ son endémicas para el Perú y una especie endémica para Canta. Asimismo, se encontró 35 especies (33.7\%) bajo amenaza: de los cuales 24 especies $(23.1 \%)$ están en peligro crítico (CR), peligro (EN) 4 especies (3.9\%) y vulnerable (VU) 7 especies (6.7\%); casi amenazadas (NT) 43 especies (41.5\%) y comunes y abundantes (LC) 25 especies $(24.0 \%)$.

Palabras clave: Flora-de-Canta, río-Chillón, especies amenazadas, endemismo, biogeografía, uso tradicional.
\end{abstract}

\begin{abstract}
Conservation status of 104 species of traditional use from the Province of Canta, Lima were determined. This study was performed during 2003, 2004 and 2005, following the CDC (1991) and UICN (1998 \& 2002) methodologies modified according to the requirements of the region and national conditions of Peru. The variables taken into consideration were: geographic distribution, abundance, antiquity of collections, location in exposed areas, extrativism, endemism, confinement; presence in conservation and protection units in situ. The results show that 22 species $(21,2 \%)$ are endemic for Peru and one is endemic for Canta. The following was also found: 35 are threatened species (33.7\%) of which 24 species (23.1\%) are in critical danger (CR), 4 species in danger (IN) (3.9\%) and 7 species are in vulnerable condition (VU) (6.7\%); 43 species are almost threatened (NT) (41.5\%) and 25 species are common and abundant (LC) $(24.0 \%)$.
\end{abstract}

Key words: Flora of Canta, Chillon River, threatened species, endemism, biogeography, traditional use.

\section{Introducción}

El Perú se localiza en América del Sur, hacia la costa occidental del pacífico, está compuesto por ecosistemas altamente fragmentados, debido a gradientes altitudinales marcados que producen cambios en la temperatura, precipitación, humedad y suelos; sin embargo, el impacto antrópico es uno de los principales modificadores de su ecología (Dillon et al., 1995; Gentry, 1982; Young, 1991; Weberbauer, 1945; Brack, 2004). En la actualidad, los hábitats andinos presentan el más alto grado de fragmentación, de allí la necesidad y prioridad para su conservación (Chepstow-Lusty \& Winfied, 2000; Young, 1994; Fahrig, 2003); Sin embargo existen pocos estudios realizados en estos aspectos, por lo que deben impulsarse investigaciones en todas las líneas a fin de tomar decisiones inmediatas en cuanto a la protección y al manejo adaptativo de los recursos (Berkes, 2004;
Chepstow-Lusty \& Winfied, 2000; Pandey, 2003; INRENA, 2004).

El presente trabajo se realizó en Canta, comprendida entre las coordenadas geográficas

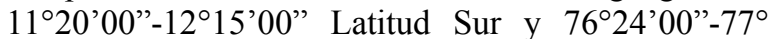
10 '00" Longitud Oeste, entre una altitud de 600-4800 msnm, con una superficie de $1687 \mathrm{Km}^{2}$ aproximadamente. De clima variado, con temperaturas que van desde $19.9^{\circ} \mathrm{C}$ en parte más baja, hasta los 5 ${ }^{\circ} \mathrm{C}$ en las más altas, y precipitaciones totales entre $18.2 \mathrm{~mm} / \mathrm{año}$, en la parte baja y $1000 \mathrm{~mm} / \mathrm{año}$ en las cumbres (SEDAPAL, 1997). La población compuesta por 10996 habitantes, siendo el $60 \%$ andina. La economía está sustentada principalmente por la agricultura, ganadería y últimamente el turismo ecológico.

Estudios botánicos son escasos, sin embargo, se reportan colecciones de Hipólito Ruiz López y José Antonio Pavón quienes recorrieron la parte central de 


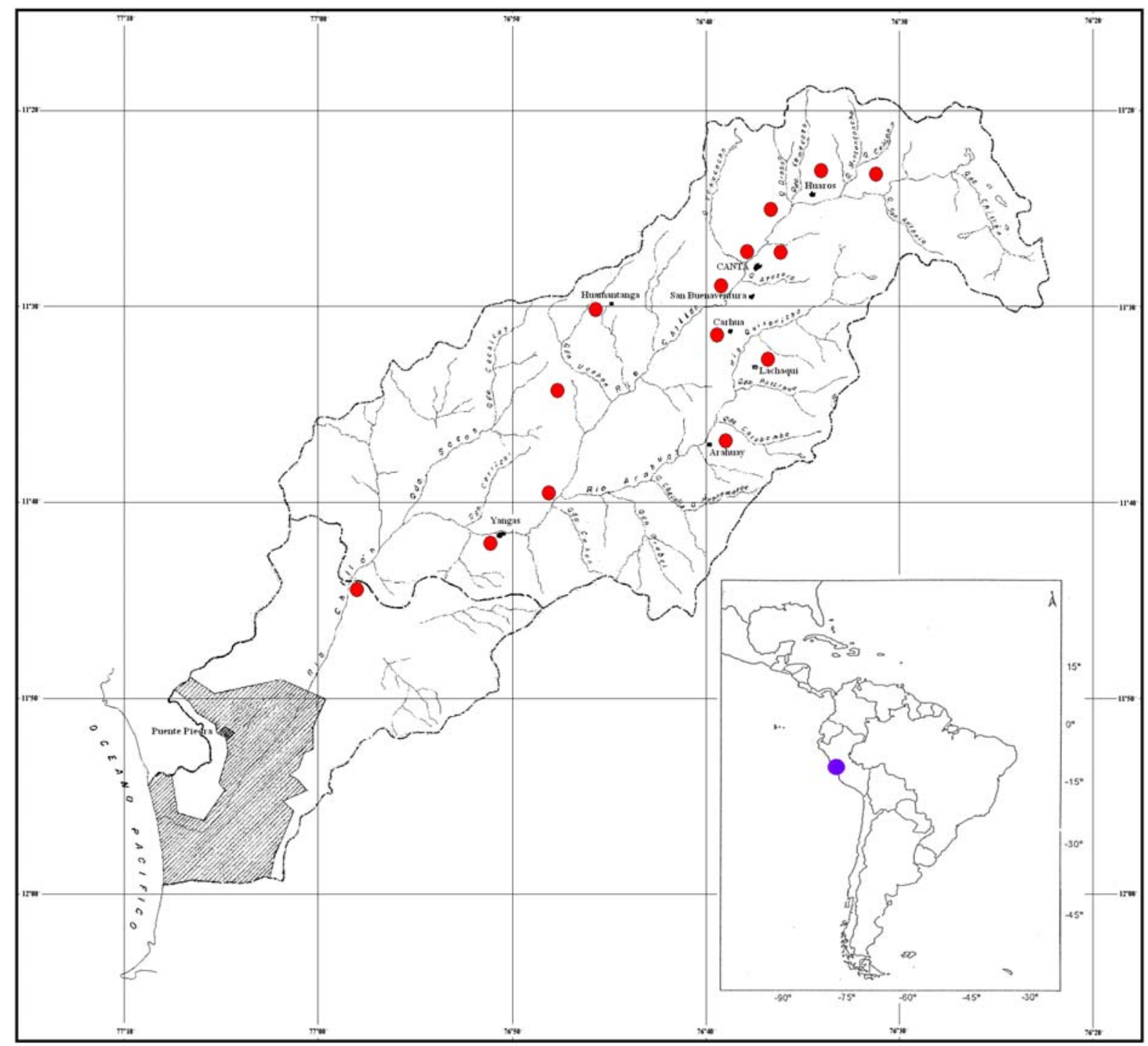

Figura 1. Localización de áreas de muestreo ( • ) en la provincia de canta y ubicación del área en estudio respecto el Perú y América Latina

las vertientes occidentales y orientales de los Andes peruanos entre los años 1777-1788, colecciones depositadas en el Herbario del Real Jardín Botánico de Madrid (RJB); colecciones de la expedición Wilkes (1818) se encuentran en el Herbario Nacional de USA y los duplicados en el Herbario ASA GRAY de Cambridge (Vilcapoma, 1987). También, existen colecciones de Augusto Weberbauer en los Herbarios de la Universidad Nacional Agraria La Molina (MOL) y del Museo de Historia Natural de la Universidad Nacional Mayor de San Marcos (USM), reportadas en el "El mundo Vegetal de los Andes Peruanos" Weberbauer (1945), donde incluye colecciones de la zona. "Flora of Peru" de Francis Macbride (1936) reporta colecciones de la sierra peruana, chequeadas y ampliadas por Bracko \& Zurucchi (1993) en "Catalogue of the Flowering Plants and Gymnosperms of Perú" último inventario de la flora peruana donde considera especies de Canta, abordando de manera referencial el status de conservación.

Investigaciones botánicas específicamente para Canta, fueron realizadas por Vilcapoma (1987) quien estudió 57 especies de la familia Solanaceae; además reporta plantas tóxicas para la zona (Vilcapoma, 2001); las Cactáceas por Teixeira et al., (2004); asimismo, Flores (1997) describe 48 especies de la familia Fabaceae.

Evaluación sobre la situación poblacional de las especies vegetales de la Cuenca del río Chillón es escasa; sin embargo, existen estudios como el de Bracko \& Zarucchi (1993) en "Catalogue of the Flowering Plants and Gymnosperms of Perú", inventario de la flora peruana, donde incluye especies de la flora de Canta, abordando de manera referencial, el status de conservación. Así mismo Flores (1997) considera a Senna malaspinae como endémica para el 
valle del Chillón lo que no encontró registros en otras localidades del país.

La presente investigación tiene por objetivo determinar el status de conservación de las especies silvestres de uso tradicional de Canta, en función a la metodología propuesta por la Unión Internacional para la Conservación de la Naturaleza (1998-2002) y la del Centro de Datos para la Conservación de la Naturaleza (1991), que permita tomar decisiones en protección y manejo.

\section{Material y Métodos}

El estudio se realizó en la provincia de Canta, la que se encuentra ubicada al este de la Provincia de Lima en la Región Lima (Figura 1), donde se muestreo 14 localidades, desde Abril 2003 a Marzo 2005; asimismo, revisaron y chequearon un total de 10449 registros de exsicatas de los herbarios: USMUniversidad Mayor de San Marcos (496), MOLUniversidad Nacional Agraria La Molina (683), UTUniversidad Nacional de Trujillo (228), HAOUniversidad Privada Antenor Orrego (293), PRGUniversidad Nacional Pedro Ruiz Gallo (78); también, los sites: MO-Missouri Botanical Garden y NYBGNew York Botanical Garden (7957); además, bibliografía especializada (714) (Bracko \& Zarucchi, 1993; Macbride, 1936; Weberbauer, 1945; Sagástegui et al., 1995; Sánchez \& Briones, 1992; Ferreyra, 1986; Vilcapoma, 1987; Flores, 1997; Tovar, 1993; López, 1998; Mostacero et al., 2002 ).

La vegetación nativa estudiada se encuentra por lo general en bosquetes bajos, caminos de herradura, chacras en barbecho o abandonadas, así como en cerco vivo

La determinación del status de conservación o situación poblacional se realizó para 104 especies en función a la metodología del Centro de Datos para la Conservación (1991) y UICN (1998 \& 2002), modificada para las condiciones de la región y del país. Se consideró: distribución geográfica, abundancia y antigüedad de colecciones, localización en áreas expuestas, extrativismo, endemismo, confinamiento, presencia en unidades de conservación y protección in situ. Las categorías consideradas fueron: extinguida (EX); extinguida en estado silvestre (EW); en peligro crítico, que enfrenta riesgo muy extremo de extinción en estado silvestre en un futuro inmediato (CR); en peligro, enfrenta un alto riesgo de extinción o deterioro poblacional en estado silvestre en un futuro próximo (EN); vulnerable (VU); amenazada, que puede calificarse como vulnerable en un futuro próximo (NT); menor preocupación o sin peligro, comunes y abundantes (LC); sin datos suficientes para ser evaluada (DD), y no evaluada (NE).

\section{Resultados y Discusión}

Se reportó, para Canta, la ocurrencia de 104 especies silvestres de uso, de las cuales se revisaron y chequearon 10449 registros de exsicatas (Tabla 1).

Tabla 1. Número de registros de las especies en tres fuentes de consulta

\begin{tabular}{lcc}
\hline \multirow{2}{*}{ Fuentes de consulta } & \multicolumn{2}{c}{ Registros } \\
\cline { 2 - 3 } & Número & $\begin{array}{c}\text { Porcentaje } \\
(\%)\end{array}$ \\
\hline Herbarios & 1778 & 17.02 \\
Sites de Internet & 7957 & 76.15 \\
Bibliografía de referencia & 714 & 6.83 \\
\hline
\end{tabular}

De las 104 especies registradas con uso tradicional en Canta, 22 especies (21.2\%) son endémicas para el Perú aunque con distribución en otras regiones andinas del país, y una especie endémica para la cuenca como es el caso de Senecio cantensis; las demás especies se encuentran distribuidas en otros países principalmente de América Latina (Tabla 2).

Tabla 2. Número de especies endémicas para el Perú.

\begin{tabular}{lcc}
\hline \multirow{1}{*}{ Endemismo } & \multicolumn{2}{c}{ Especies } \\
\cline { 2 - 3 } & Número & $\begin{array}{c}\text { Porcentaje } \\
(\%)\end{array}$ \\
\hline Endémicas de Perú & 22 & 21.15 \\
No endémicas & 81 & 77.88 \\
Sin datos suficientes & 1 & 0.96 \\
\hline
\end{tabular}

Se verificó la escasez de colecciones de las 104 especies estudiadas: 755 exsicatas $(7.2 \%)$ para Canta, 2850 exsicatas $(27.3 \%)$ para el resto del país; sin embargo, 6844 exsicatas $(65.5 \%)$ fueron reportadas en el extranjero, incluyéndose material colectado en el Perú cuyos registros se encuentran en: Bracko \& Zurucchi (1993); Gentry \& Forsyth (1998); Macbride (1936), por lo que es necesario ampliar e intensificar las recolecciones en la zona de estudio y en otras regiones andinas que ayuden a categorizar adecuadamente cada especie (Anexo 1).

La antigüedad de las colecciones encontradas de las muestras botánicas en los 5 herbarios, registro de 2 sites de la Internet y bibliografía especializada determinaron que: 82 especies $(78.9 \%)$ fueron colectadas antes de 1990, 20 especies (19.3\%) entre $1990-2000$ y 2 especies (1.9\%) después de 2000. Situación, que indica evidentemente la escasez de información actualizada, lo que implica, tomando en cuenta el párrafo anterior y la metodología de la UICN (1998; 2002) la necesidad de incluirlas en categorías 
de mayor riesgo, como principio de predicción, para la conservación de las especies (Anexo 1).

En cuanto a la distribución mundial de las especies se reportó que 70 especies $(67.31 \%)$ se encuentran en menos de tres países, por lo general pertenecientes a la subregión andina; 19 especies (18.27\%) entre 4-9 países básicamente de la región Neotropical, y 14 especies $(13.46 \%)$ se encuentran ampliamente distribuidos en más de 10 países con carácter cosmopolita, como por ejemplo: Asclepias curassavica, Chenopodium ambrosioides, Chamaesyce hirta, Mikania micrantha, entre otras, y una especies con poca información como es el caso de Sessea confertiflora.

En el Perú la distribución de las especies se reportan principalmente en los departamentos que cuentan con contrafuertes andinos como Puno, Cusco, Apurímac, Lima, Cajamarca, La Libertad, Pasco entre los más importantes. 40 especies $(38.76 \%)$ ocurren en menos de tres departamentos, 62 especies (59.62\%) entre 4 y 10 departamentos, que comprenden ecosistemas de costa y sierra, y $2(1.92 \%)$ en casi todos los departamentos, inclusive en aquellos con ecosistemas húmedos tropicales coincidente con estudios semejantes (Bracko \& Zurucchi, 1993; Sagástegui \& Leiva, 1993; Sánchez \& Briones, 1992; Vilcapoma, 1987; Flores, 1997; Tovar, 1993; López, 1998; Gentry \& Forsyth, 1998; Gentry, 1982; Cerrate, 1979; INRENA, 1995) (Anexo 1).

Del total, 11 especies $(10.58 \%)$ se localizan en áreas expuestas y 93 especies $(89.42 \%)$ en áreas más restringidas; sin embargo, las especies se encuentran en cercos vivos, bordes de chacras, borde de caminos, base de andenes antiguos, así como en áreas de barbecho agropecuario en valles interandinos, laderas y punas, donde están localizadas las zonas expuestas a una intensa actividad agrícola y ganadera, y sumado a la escasez de leña y la inestabilidad del suelo debido a las condiciones fisiográficas, muchas de ellas están desapareciendo progresivamente (INRENA, 2004; Dillon et al., 1995; Young, 1991; CDC, 1991; Chepstow-Lusty \& Winfield, 2000; Valencia et al., 2000; Sagástegui \& Leiva, 1993; Sánchez \& Briones, 1992; Cerrate, 1980; Vilcapoma, 2001).

De las 104 especies se aprovechan las hojas, flores, frutos, tallos, cortezas, y muchas veces la raíz y rizomas lo que implica daños severos a la planta ocasionando su muerte, sin reparar que su propagación es espontánea, no habiendo un aprovechamiento y/o manejo adecuado como también ocurre en otros lugares (Martínez et al., 2000; Alarcón \& Mena, 1994; Köhler et al., 2005; Muñoz, 1987; Conner, 2001; Morales, 1996; Miraldi et al., 2001). Se ha determinado 14 especies (13.46) con alto grado de extrativismo como Equisetum bogotense, Krameria lappacea, Muehlenbeckia volcanica, Perezia coerulescens, Senecio canescens, Senecio rhizomatus, Plantago major, Gnaphalium lacteum, entre otras; 36 especies (34.62\%) con nivel medio como: Acanthoxanthium spinosum, Ageratina stembergiana, Aristeguietia discolor; 42 especies (40.38\%) baja, cuyo aprovechamiento es solo de hojas y/o flores, y 12 especies (11.54\%) sin datos suficientes que dificulta la categorización en este aspecto (Anexo 1). El cultivo de plantas medicinales en la zona no es practicado por los agricultores tradicionales, las mejores tierras están dedicadas a los cultivos agrícolas y pastos, mientras que las plantas medicinales se colectan de su estado silvestre. Sin embargo el cultivo de plantas medicinales es de gran potencial económico para las zonas pobres de la provincia de Canta como también lo indican Martínez et al. (2000); Muñoz, (1987); Harsha et al. (2002); Cáceres, 1991; Conner, 2001; Gary, 1995; Hammond et al, 1998).

En cuanto a la distribución de las especies en áreas relativamente pequeñas se encontró que 16 especies $(15.38 \%)$ están confinadas en el sentido longitudinal y 3 especies $(2.88 \%)$ en el sentido latitudinal, colocándolas en una situación de extrema vulnerabilidad y que puede resultar en su extinción si sus hábitats continúan siendo destruidas por la acción antrópica y una especies sin datos suficientes para su análisis como la categorizan la UICN (1998), CDC (1991), Zavala \& Zevallos (1996) y Valencia et al. (2000) (Anexo 1).

La probabilidad de encontrar las especies silvestres de Canta en el sistema de unidades de conservación muestra ciertas variaciones, siendo 35 especies (33.65\%) con menos del $25 \%$ de probabilidad; 44 especies $(42.31 \%)$ entre 25 y $50 \%$ y solamente 25 especies $(24.04 \%$ ) con más del $50 \%$ de probabilidad de estar protegidas en las unidades de conservación del Perú; sin embargo a pesar de encontrarlas en áreas protegidas están bajo la amenaza constante de invasiones por agricultores migratorios, explotación ilegal, incendios como también lo comentan Berkes (2004); Fahring (2003); Pandey (2003) y se comprobó con el trabajo de campo.

Los resultados del análisis de correlación obtenidos en el anexo 1 y anexo 1 involucra diferentes parámetros como: (i) número de registros de las especies, (ii) antigüedad de las colecciones botánicas, (iii) localización en áreas expuestas, (iv) extrativismo, (v) características de endemismo, (vi) confinamiento de especies, (vii) presencia en unidades de conservación y presencia en otros países. 35 especies fueron encontradas bajo amenaza: 24 especies $(23.08 \%)$ en peligro crítico (CR), 4 especies $(3.85 \%)$ en peligro (EN) y 7 especies $(6.73 \%)$ vulnerable; 43 especies (41.35\%) casi amenazadas (NT); 25 especies (24.04\%) con preocupación menor (LC) y una (1) especies sin datos suficientes (DD).

Los resultados obtenidos del diagnóstico del status de las especies usadas tradicionalmente por los pobladores de Canta y que se hizo a través de revisión bibliográfica, revisión de herbarios, Sites de Internet y 
trabajo de campo, no son necesariamente categorías fijas, pueden variar por factores ambientales, sociales, así como en la medida que se disponga de mayor información. Es necesario en el Perú establecer un método general, unificado y coherente para determinar las categorías de status de las especies silvestres donde intervengan instituciones públicas y privadas que tengan que ver con la actividad de la conservación de la biodiversidad.

\section{Conclusiones y Recomendaciones}

Se determinó 22 especies endémicas para el Perú, siendo sólo Senecio cantensis endémica para la Cuenca del Río Chillón. También se encontró 14 especies comospolitas: Asclepias curassavica, Chenopodium ambrosioides, Chamaesyce hirta, Mikania micrantha entre otras.

Existen 14 especies con alto grado de extrativismo: Equisetum bogotense, Krameria lappacea, Muehlenbeckia volcanica, Perezia coerulescens, Senecio canescens, Senecio rhizomatus, Plantago majo y Gnaphalium lacteum; 36 especies (34.62\%) nivel medio; 42 especies (40.38\%) nivel bajo. Confinadas en el sentido longitudinal 16 especies $(15.38 \%)$ y confinadas en sentido latitudinal 3 especies $(2.88 \%)$.

Se encontró 35 plantas (33.65\%) bajo amenaza: de los cuales 24 especies $(23.08 \%)$ están en peligro crítico (CR), peligro (EN) 4 especies (3.85\%) y vulnerable (VU) 7 especies (6.73\%); casi amenazadas (NT) 43 especies $(41.35 \%)$ y comunes y abundantes (LC) 25 especies $(24.04 \%)$.

Debe continuarse con estudios de evaluación del status de conservación de otras especies en el valle del Chillón y se haga extensiva a otros valles del Perú

Debe establecerse en el Perú un método general, unificado y coherente para determinar las categorías de status de las especies silvestres donde intervengan instituciones públicas y privadas involucradas con la actividad de la conservación de la biodiversidad.

Debe de iniciarse estudios de agrotecnología de las especies estudiadas que nos permitan determinar su plan de cultivo para la producción de los elementos que contengan los alcaloides promisorios. Lo que permitirá evitar la migración de la población a la capital.

\section{Agradecimientos}

A los pobladores de la provincia de Canta; al Dr. Marcel Gutiérrez Correa por sus sugerencias, A los curadores de los herbarios consultados: USM, MOL, UT, HAO y PRG por su apoyo en revisión de las exsicatas de las especies estudiadas y a INCAGRO (Innovación y Competitividad para el Agro Peruano) por el financiamiento de la presente investigación.

\section{Literatura citada}

Alarcón R. \& Mena P. 1994 Etnobotánica, valoración económica y comercialización de recursos florísticos silvestres en el alto Napo-Ecuador. Ecociencia 161-162.

Berkes F. 2004. Rethinking community-based conservation. Conservation Biology. 18(3): 621-630.

Brack E. A. 1994. Posibilidades de las plantas nativas para el control de plagas, pp.3-25 In: Gomero, L. (ed). Plantas para curar cultivos. Lima, Perú.

Brako L. \& Zarucchi J. 1993. Catalogue of the Flowering Plants and Gymnosperms of Peru. Missouri Botanical Garden. Missouri-EEUU.

Cáceres A. 1991. Industrial Utilization of Indigenous Medicinal Plans for Rural Primary Health Care Systems in Guatemala. Entwickelun ländlicher raum. (91)4: 1417.

Centro de datos para la conservación-CDC. 1991. Plan Director del Sistema Nacional de Unidades de Conservación (Sinuc): Una aproximación desde la diversidad biológica. Universidad Nacional Agraria La Molina. Lima-Perú.

Cerrate E. 1980. Plantas que curan las heridas del hombre y los animales. Boletín de Lima. 4: 8-14.

Cerrate E. 1979. Vegetación del valle de Chiquian. Universidad Nacional Mayor de San Marcos. Lima, Perú.

Conner M. 2001. Economic botany. In: Plants in our world. McGraw-Hill Companies. $3^{\text {rd }}$ edition.

Chepstow-Lusty A. \& Winfield M. 2000. Inca Agroforestry: Lessons from the past. Ambio. 29(6): 322-328.

Dillon M., Sagástegui A., Sánchez I., Llatas S. \& Hensold A. 1995. Floristic Inventory and Biogeographic Analysis of Montane Forests in North-western Perú. In: Biodiversity and Conservation of Neotropical Montane Forest. The New York Botanical Garden. USA. : 251269.

Fahring L. 2003. Effects of habitat fragmentation on biodiversity. Annual review of Ecology, Evolution, and Systematics. 34: 487-515.

Ferreyra R. 1986. Flora de Perú. Lima, Universidad Nacional Mayor de San Marcos. Lima-Perú.

Flores M. 1997. La familia leguminosae en el valle del Chillón-parte media y alta, Departamento de Lima. Tésis Magíster. Universidad Nacional Mayor de San Marcos. Lima.

Gary M. 1995. Etnobotánica. Manual de Métodos. $1^{\text {er }}$ edición. Fondo Mundial Para la Naturaleza-WWF. Uruguay.

Gentry A. 1982. Neotropical floristic diversity: phytogeographical connections between Central and South America, Pleistocene climatic fluctuations, or an accident of the Andean orogeny. Annals of the Missouri Botanical Garden. 69: 557-593.

Gentry A. \& Forsyth A. 1998. A field guide to the families and genera of woody plants of northwest South America: (Colombia, Ecuador, Perú). Conservation International Washington DC. IV. (Note Complementary).

Hammond G., Fernández I., Villegas L. \& Vaisberg A. 1998. A survey of traditional medicinal plants from the Callejón de Huaylas, Department of Ancash, Perú. Journal of Ethnopharmacology .61: 17-30. 
Harsha V., Hebbar S., Hedge G. \& Shripathi V. 2002. Ethnomedical knowledge of plants used by Kunabitribe of Karnataka in India. Fitoterapia. 73: 281-287.

Instituto Nacional de Recursos Naturales-INRENA. 2004. Perú Forestal en números año 2002. INRENA. LimaPerú.

Instituto Nacional de Recursos Naturales-INRENA. 1995. Mapa ecológico del Perú: Guía explicativa, INRENA, Lima. Perú.

Instituto Geográfico Nacional-IGN.1993. Carta Nacional. Hojas: 23j, 23k, 24i, 24j. Lima.

International Union For Conservation Of Nature-UICN 1998.The IUCN Red list of threatened plants. Gland. Switzerland: IUCN,. 1v.

International Union For Conservation Of Nature-UICN. 2002. The IUCN Red list of threatened plants. Version 3.1. Gland. Switzerland: IUCN,. 1v. (species survival comisión).

Köhler B., Gigon A., Edwards P., Krüsi B., Langenauer R., Lüscher A. \& Ryser P. 2005. Changes in the species composition and conservation value of limestone grasslands in Northern Switzerland after 22 years of contrasting managements. Perspectives in Plant Ecology, Evolution and Systematics. 7: 51-67.

López A. 1998. Catálogo de la Flora del Departamento de la Libertad. Arnaldoa. 5(1): 93-126.

Macbride F. 1936. Flora of Peru. Chicago, Field Museum Natural History. V. 13.

Martínez A., Bernal Y. \& Cáceres A. 2000. Fundamentos de agrotecnología de cultivo de plantas medicinales Iberoamericanas. Santafé de Bogotá, Colombia Convenio Andrés Bello/Ciencia y Tecnología para el Desarrollo.

Miraldi E., Ferri S. \& Mostaghimi V. 2001. Botanical drug and preparations in the traditional medicine of West Azerbaijan, Iran. Journal of Ethnopharmacology. 75: 77 87.

Morales R. 1996. Farmacología y Farmacognosia como fuentes de validación y contraste en etnobotánica. Jardín Botánico de CordoBa. Argentina. 3: 93-98.

Mostacero J., Mejía F. \& Gamarra O. 2002. Taxonomía de las Fanerógamas útiles del Perú. Ed. Normas Legales. Trujillo, Perú.
Muñoz F. 1987. Plantas medicinales y aromáticas. Estudio, cultivo y procesado. Ed. Mundi. Madrid.

Pandey D. 2003. Cultural resources for conservation science. Conservation Biology. 17(2): 633-635.

Sánchez I. \& Briones A. 1992. Nombres vulgares de las especies vegetales de la ladera del valle de Cajamarca. Cajamarca, Perú.

Sagástegui A. \& Leiva S. 1993. Flora invasora de los cultivos del Perú. Ed. Libertad EIRL. Trujillo. Perú.

Servicio de Agua Potable y Alcantarillado de Lima SEDAPAL-Banco Interamericano de Reconstrucción y Fomento (BIRF). 1997. Estudio de Factibilidad del Desarrollo para el aprovechamiento optimo de las aguas superficiales y subterráneas del rió Chillón, Servicio de Agua Potable y Alcantarillado de Lima. Lima-Perú.

Teixeira V., Castro V., Ceroni A. \& Eyzaguirre R. 2004 Diversidad y densidad de la Comunidad de Cactáceas en el cerro Umarcata y quebrada oropel en el valle del río Chillón (Lima) y su relación con los factores edáficos. Ecol. Apl. 3 (1 y 2): 1-8

Tovar O. 1993. Las gramíneas (Poaceae) del Perú. Monografías del Real Jardín Botánico. T(13), Ruiza, Madrid.

Valencia R., Pitman N., León-Yánez S. \& Jorgensen P. 2000. Libro rojo de las plantas endémicas del Ecuador 2000. Herbario QCA, Pontificia Universidad Católica del Ecuador, Quito.

Vilcapoma G. 1987. Las solanaceas del valle del Chillón. Boletín de Lima. 52: 63-82. 2001. Plantas tóxicas de la Cuenca del río Chillón. Biota. 100: 71-79.

Weberbauer A. 1945. El mundo vegetal de los Andes Peruanos. Estación. Experimental Agrícola de la Molina. Lima, Perú.

Young K. 1991. Floristic diversity on the eastern slopes of the Peruvian Andes. Candollea. 46: 125-143.

1994. Roads and the environmental degradation of tropical montane forests. Conservation Biology. 8(4): 972-976.

Zavala A. \& Zevallos P. 1996. Taxonomía, distribución geográfica y status del género Uncaria en el Perú.

Universidad Nacional Agraria La Molina, Lima, Perú. 
Anexo 1. Status de conservación de las especies de uso tradicional en medicina y biocida en la Cuenca del rio Chillón.

(i) Número de registros; (ii) antigüedad de colectas: $\mathrm{A}=$ colectas antes de $1990, \mathrm{~B}=$ colectas entre $1990-2000, \mathrm{C}=$ colectas recientes despues del 2000; (iii) localización en áreas expuestas: $\mathrm{X}=\mathrm{intensa}$ actividad agropecuaria, $\mathrm{XX}=$ actividad agropecuaria moderada; (iv) grado de extraivismo: $\mathrm{A}=\mathrm{alta}, \mathrm{M}=$ =me(ia, $\mathrm{B}=$ baja, $\mathrm{S}=$ sin datos; (v) endemismo: $\mathrm{X}=$ =ndémicas, $\mathrm{XX}=$ no endémicas; (vi) número de paises donde se reporta la especie; (vii) confinamiento: $\mathrm{X}=$ confinada, $\mathrm{N}=$ no confinada; (viii) probabilidad de encontrarla en unidades de conservación (0..1); (ix) categorización: $\mathrm{EX}=$ extinguida, $\mathrm{EW}=$ extinguida en estado silvestre, $\mathrm{CR}=$ en peligro crítico, $\mathrm{EN}=$ en peligro, $\mathrm{VU}=$ vulnerable, $\mathrm{NT}=$ amenazada, $\mathrm{LC}=$ comunes $\mathrm{y}$ abundantes, $\mathrm{DD}=$ sin datos, $\mathrm{NE}=$ no evaluada.

\begin{tabular}{|c|c|c|c|c|c|c|c|c|c|c|c|c|c|}
\hline \multirow{3}{*}{ Especies } & \multicolumn{4}{|c|}{\begin{tabular}{|} 
Números de registros de especies \\
(i)
\end{tabular}} & \multirow{3}{*}{ 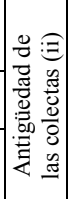 } & \multirow{3}{*}{ 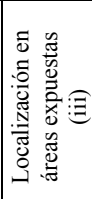 } & \multirow{3}{*}{ 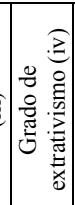 } & \multirow{3}{*}{ 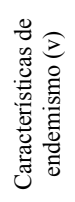 } & \multirow{3}{*}{  } & \multicolumn{2}{|c|}{$\begin{array}{c}\text { Confinamiento } \\
\text { (vii) }\end{array}$} & \multirow{3}{*}{ 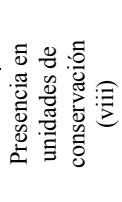 } & \multirow{3}{*}{ 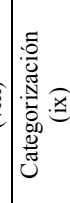 } \\
\hline & \multicolumn{2}{|c|}{ Perú } & \multirow[t]{2}{*}{$\begin{array}{c}\text { Otros } \\
\text { países }\end{array}$} & \multirow[t]{2}{*}{ Total } & & & & & & & & & \\
\hline & Canta & Otros & & & & & & & & Long. & Altit. & & \\
\hline Acanthoxanthium spinosum (L.) Fourr. & 8 & 2 & 10 & 20 & $\mathrm{C}$ & $\mathrm{X}$ & $\mathrm{S}$ & $\mathrm{N}$ & 2 & $\mathrm{~N}$ & $\mathrm{~N}$ & 0.24 & $\mathrm{LC}$ \\
\hline Acnistus arborescens (L.) Schltdl. & 12 & 90 & 138 & 240 & B & $\mathrm{xx}$ & B & $\mathrm{N}$ & 12 & $\mathrm{~N}$ & $\mathrm{~N}$ & 0.49 & $\mathrm{LC}$ \\
\hline Achyrocline alata (Kunth) DC. & 15 & 104 & 91 & 210 & A & $\mathrm{xx}$ & M & $\mathrm{N}$ & 5 & $\mathrm{~N}$ & $\mathrm{~N}$ & 0.76 & $\mathrm{LC}$ \\
\hline Agave americana $\mathrm{L}$. & 2 & 3 & 30 & 35 & A & $\mathrm{xx}$ & B & $\mathrm{N}$ & 6 & $\mathrm{~N}$ & $\mathrm{~N}$ & 0.22 & NT \\
\hline $\begin{array}{l}\text { Ageratina sternbergiana (DC.) R.M. } \\
\text { King \& H. Rob. }\end{array}$ & 12 & 48 & 27 & 87 & B & $\mathrm{XX}$ & M & $\mathrm{N}$ & 1 & $\mathrm{~N}$ & $\mathrm{~N}$ & 0.23 & NT \\
\hline Alnus acuminata Kunth & 3 & 23 & 17 & 43 & A & $\mathrm{xx}$ & B & $\mathrm{N}$ & 2 & $\mathrm{~N}$ & $\mathrm{~N}$ & 0.69 & NT \\
\hline Ambrosia arborescens Mill. & 3 & 23 & 69 & 95 & B & $\mathrm{xx}$ & B & $\mathrm{N}$ & 2 & $\mathrm{~N}$ & $\mathrm{~N}$ & 0.55 & LC \\
\hline Argemone subfusiformis G.B. Ownbey & 3 & 12 & 20 & 35 & A & $\mathrm{xx}$ & B & $\mathrm{N}$ & 3 & $\mathrm{~N}$ & $\mathrm{~N}$ & 0.53 & NT \\
\hline $\begin{array}{l}\text { Aristeguietia discolor R.M. King \& H. } \\
\text { Rob. }\end{array}$ & 15 & 34 & 0 & 49 & A & $\mathrm{xX}$ & M & $\mathrm{x}$ & 0 & $\mathrm{~N}$ & $\mathrm{~N}$ & 0.63 & NT \\
\hline Asclepias curassavica $\mathrm{L}$. & 2 & 67 & 1423 & 1492 & A & $\mathrm{xx}$ & B & $\mathrm{N}$ & 21 & $\mathrm{~N}$ & $\mathrm{~N}$ & 0.82 & $\mathrm{LC}$ \\
\hline Astragalus garbancillo Cav. & 15 & 52 & 18 & 85 & A & $\mathrm{xx}$ & S & $\mathrm{N}$ & 3 & $\mathrm{~N}$ & $\mathrm{~N}$ & 0.15 & vU \\
\hline Azorella crenata (Ruiz \& Pav.) Pers. & 4 & 24 & 23 & 51 & A & $\mathrm{xX}$ & $\mathrm{s}$ & $\mathrm{N}$ & 2 & $\mathrm{~N}$ & $\mathrm{~N}$ & 0.64 & $\mathrm{VU}$ \\
\hline Baccharis genistelloides (Lam.) Pers. & 9 & 98 & 134 & 241 & A & $\mathrm{xX}$ & M & $\mathrm{N}$ & 4 & $\mathrm{~N}$ & $\mathrm{~N}$ & 0.67 & $\mathrm{LC}$ \\
\hline Baccharis latifolia (Ruiz \& Pav.) Pers. & 9 & 104 & 165 & 278 & A & $\mathrm{XX}$ & M & $\mathrm{N}$ & 5 & $\mathrm{~N}$ & $\mathrm{~N}$ & 0.70 & $\mathrm{LC}$ \\
\hline Baccharis odorata Kunth & 17 & 23 & 12 & 52 & A & $\mathrm{xx}$ & M & $\mathrm{N}$ & 2 & $\mathrm{~N}$ & $\mathrm{x}$ & 0.16 & $\mathrm{CR}$ \\
\hline $\begin{array}{l}\text { Baccharis salicifolia (Ruiz \& Pav.) } \\
\text { Pers. }\end{array}$ & 6 & 42 & 128 & 176 & A & $\mathrm{xx}$ & M & $\mathrm{N}$ & 11 & $\mathrm{~N}$ & $\mathrm{~N}$ & 0.52 & LC \\
\hline Berberis flexuosa Ruiz \& Pav. & 4 & 12 &  & 16 & A & $\mathrm{xx}$ & M & $\mathrm{x}$ & 0 & $\mathrm{x}$ & $\mathrm{N}$ & 0.22 & $\mathrm{CR}$ \\
\hline Berberis monosperma Ruiz \& Pav. & 4 & 13 & 1 & 18 & $\mathrm{~B}$ & $\mathrm{XX}$ & M & $\mathrm{N}$ & 1 & $\mathrm{~N}$ & $\mathrm{~N}$ & 0.33 & $\mathrm{CR}$ \\
\hline Bidens pilosa $\mathrm{L}$. & 18 & 66 & 247 & 331 & B & $\mathrm{xx}$ & B & $\mathrm{N}$ & 15 & $\mathrm{~N}$ & $\mathrm{~N}$ & 0.52 & LC \\
\hline Kuntze & $\begin{array}{c}3 \\
13\end{array}$ & $\begin{array}{l}67 \\
51\end{array}$ & $\begin{array}{l}36 \\
68\end{array}$ & $\begin{array}{l}106 \\
132\end{array}$ & $\begin{array}{l}\mathrm{A} \\
\mathrm{A}\end{array}$ & $\begin{array}{l}\mathrm{XX} \\
\mathrm{XX}\end{array}$ & $\begin{array}{l}\mathrm{B} \\
\mathrm{B}\end{array}$ & $\begin{array}{l}\mathrm{N} \\
\mathrm{N}\end{array}$ & $\begin{array}{c}13 \\
4\end{array}$ & $\begin{array}{l}\mathrm{N} \\
\mathrm{N}\end{array}$ & $\begin{array}{l}\mathrm{N} \\
\mathrm{N}\end{array}$ & 80 & $\begin{array}{l}\text { NT } \\
\text { LC }\end{array}$ \\
\hline & 7 & 18 & 0 & 25 & A & $\mathrm{X}$ & $\mathrm{B}$ & $\mathrm{X}$ & 0 & $\mathrm{X}$ & $\mathrm{N}$ & & $\mathrm{CR}$ \\
\hline Calceolaria bicolor Ruiz & 20 & 15 & 0 & 35 & B & $\mathrm{XX}$ & B & $\mathrm{X}$ & 0 & $\mathrm{X}$ & $\mathrm{N}$ & 0.35 & NT \\
\hline Calceolaria lobata $\mathrm{Cav}$. & 13 & 19 & 4 & 36 & A & $\mathrm{xx}$ & B & $\mathrm{N}$ & 1 & $\mathrm{~N}$ & $\mathrm{~N}$ & 0.36 & NT \\
\hline Cestrum auriculatum L'Hér. & 5 & 32 & 14 & 51 & A & $\mathrm{xx}$ & B & $\mathrm{N}$ & 1 & $\mathrm{~N}$ & $\mathrm{~N}$ & 0.45 & NT \\
\hline Conium maculatum $\mathrm{L}$. & 6 & 17 & 85 & 108 & A & $\mathrm{xX}$ & $\mathrm{s}$ & $\mathrm{N}$ & 8 & $\mathrm{~N}$ & $\mathrm{~N}$ & 0.46 & NT \\
\hline Chamaesyce hirta (L.) Millsp. & 5 & 21 & 493 & 519 & B & $\mathrm{xX}$ & B & $\mathrm{N}$ & 17 & $\mathrm{~N}$ & $\mathrm{~N}$ & 0.31 & $\mathrm{LC}$ \\
\hline Chamaesyce hypericifolia (L.) Millsp. & 18 & 23 & 292 & 333 & A & $\mathrm{xx}$ & B & $\mathrm{N}$ & 13 & $\mathrm{~N}$ & $\mathrm{~N}$ & 0.15 & LC \\
\hline Chenopodium ambrosioides $\mathrm{L}$. & 4 & 75 & 380 & 459 & B & $\mathrm{xx}$ & B & $\mathrm{N}$ & 21 & $\mathrm{~N}$ & $\mathrm{~N}$ & 0.48 & LC \\
\hline Chuquiraga spinosa Less. & 21 & 54 & 0 & 75 & A & $\mathrm{xx}$ & B & $\mathrm{x}$ & 0 & $\mathrm{x}$ & $\mathrm{N}$ & 0.47 & VU \\
\hline $\begin{array}{l}\text { Descurainia myriophylla (Willd. ex } \\
\text { DC.) R.E. Fr. }\end{array}$ & 5 & 31 & 20 & 56 & A & $\mathrm{xx}$ & B & $\mathrm{N}$ & 3 & $\mathrm{~N}$ & $\mathrm{~N}$ & 0.48 & NT \\
\hline Dicliptera peruviana (Lam.) Juss. & 5 & 20 & 9 & 34 & A & $\mathrm{xX}$ & B & $\mathrm{N}$ & 1 & $\mathrm{~N}$ & $\mathrm{~N}$ & 0.45 & NT \\
\hline Drymaria grandiflora Bartl. & 8 & 15 & 1 & 24 & B & $\mathrm{xx}$ & B & $\mathrm{N}$ & 1 & $\mathrm{~N}$ & $\mathrm{~N}$ & 0.25 & NT \\
\hline Equisetum bogotense Kunth & 4 & 51 & 252 & 307 & A & $\mathrm{xx}$ & A & $\mathrm{N}$ & 7 & $\mathrm{~N}$ & $\mathrm{~N}$ & 0.17 & EN \\
\hline $\begin{array}{l}\text { Erodium cicutarium (L.) L'Hér. ex } \\
\text { Aiton }\end{array}$ & 10 & 19 & 86 & 115 & A & $\mathrm{xX}$ & M & $\mathrm{N}$ & 6 & $\mathrm{~N}$ & $\mathrm{~N}$ & 0.43 & NT \\
\hline Foeniculum vulgare Mill. & 5 & 13 & 47 & 65 & B & $\mathrm{xx}$ & M & $\mathrm{N}$ & 8 & $\mathrm{~N}$ & $\mathrm{~N}$ & 0.26 & NT \\
\hline Fuertesimalva peruviana $(\mathrm{L}$.$) Fryxell$ & 7 & 16 & 0 & 23 & B & $\mathrm{xx}$ & M & $\mathrm{x}$ & 0 & $\mathrm{x}$ & $\mathrm{N}$ & 0.30 & $\mathrm{CR}$ \\
\hline Fumaria capreolata $\mathrm{L}$. & 6 & 23 & 6 & 35 & B & $\mathrm{xx}$ & B & $\mathrm{N}$ & 2 & $\mathrm{~N}$ & $\mathrm{~N}$ & 0.16 & NT \\
\hline Furcraea occidentalis Trelease & 3 & 18 & 1 & 22 & A & $\mathrm{xx}$ & M & $\mathrm{N}$ & 1 & $\mathrm{~N}$ & $\mathrm{~N}$ & 0.24 & $\mathrm{CR}$ \\
\hline Gamochaeta purpurea (L.) Cabrera & 4 & 30 & 88 & 122 & A & $\mathrm{xX}$ & $\mathrm{M}$ & $\mathrm{N}$ & 4 & $\mathrm{~N}$ & $\mathrm{~N}$ & 0.65 & $\mathrm{LC}$ \\
\hline Gnaphalium lacteum Meyen \& Walp. & 8 & 11 & 2 & 21 & A & $\mathrm{xx}$ & $\mathrm{s}$ & $\mathrm{N}$ & 1 & $\mathrm{~N}$ & $\mathrm{~N}$ & 0.23 & NT \\
\hline $\begin{array}{l}\text { Hypochaeris taraxacoides (Meyen \& } \\
\text { Walp.) Ball }\end{array}$ & 5 & 11 & 18 & 34 & A & $\mathrm{xx}$ & M & $\mathrm{N}$ & 2 & $\mathrm{~N}$ & $\mathrm{~N}$ & 0.20 & NT \\
\hline $\begin{array}{l}\text { Iochroma umbellatum (Ruiz \& Pav.) } \\
\text { Hunziker ex D'Arcy }\end{array}$ & 4 & 23 & 0 & 27 & A & $\mathrm{xX}$ & $\mathrm{s}$ & $\mathrm{x}$ & 0 & $\mathrm{x}$ & $\mathrm{N}$ & 0.32 & $\mathrm{CR}$ \\
\hline Jaltomata bicolor (Ruiz \& Pav.) Mione & 9 & 15 & 0 & 24 & A & $\mathrm{x}$ & B & $\mathrm{x}$ & 0 & $\mathrm{x}$ & $\mathrm{N}$ & 0.20 & $\mathrm{CR}$ \\
\hline Jungia paniculata (DC.) A. Gray & 13 & 47 & 7 & 67 & A & $\mathrm{xx}$ & B & $\mathrm{N}$ & 1 & $\mathrm{~N}$ & $\mathrm{~N}$ & 0.28 & NT \\
\hline Justicia sericea Ruiz \& Pav. & 4 & 20 & 4 & 28 & A & $\mathrm{XX}$ & S & $\mathrm{N}$ & 1 & $\mathrm{~N}$ & $\mathrm{~N}$ & 0.48 & NT \\
\hline $\begin{array}{l}\text { Krameria lappacea (Dombey) Burdet \& } \\
\text { B.B. Simpson }\end{array}$ & 2 & 33 & 10 & 45 & A & $\mathrm{xx}$ & A & $\mathrm{N}$ & 3 & $\mathrm{~N}$ & $\mathrm{~N}$ & 0.54 & $\mathrm{CR}$ \\
\hline Lantana zahlbruckneri Hayek & 6 & 8 & 0 & 14 & A & $\mathrm{xx}$ & M & $\mathrm{x}$ & 0 & $\mathrm{x}$ & $\mathrm{N}$ & 0.50 & $\mathrm{CR}$ \\
\hline Lepechinia meyenii (Walp.) Epling & 2 & 18 & 7 & 27 & A & $\mathrm{xx}$ & M & $\mathrm{N}$ & 2 & $\mathrm{~N}$ & $\mathrm{~N}$ & 0.48 & NT \\
\hline
\end{tabular}




\begin{tabular}{|c|c|c|c|c|c|c|c|c|c|c|c|c|c|}
\hline Lobelia decurrens Cav. & 7 & 21 & 17 & 45 & $\mathrm{C}$ & $\mathrm{XX}$ & $\mathrm{B}$ & $\mathrm{N}$ & 2 & $\mathrm{~N}$ & $\mathrm{~N}$ & 0.15 & $\mathrm{VU}$ \\
\hline Lycopersicon hirsutum Dunal & 10 & 29 & 16 & 55 & B & $\mathrm{XX}$ & B & $\mathrm{N}$ & 1 & $\mathrm{~N}$ & $\mathrm{~N}$ & 0.25 & NT \\
\hline Marrubium vulgare $\mathrm{L}$. & 3 & 26 & 96 & 125 & A & $\mathrm{XX}$ & $\mathrm{S}$ & $\mathrm{N}$ & 5 & $\mathrm{~N}$ & $\mathrm{~N}$ & 0.49 & $\mathrm{LC}$ \\
\hline Mikania micrantha Kunth & 7 & 69 & 451 & 527 & A & $\mathrm{XX}$ & $\mathrm{B}$ & $\mathrm{N}$ & 14 & $\mathrm{~N}$ & $\mathrm{~N}$ & 0.71 & $\mathrm{LC}$ \\
\hline Minthostachys mollis (Kunth) Griseb. & 14 & 53 & 42 & 109 & A & $\mathrm{XX}$ & M & $\mathrm{N}$ & 4 & $\mathrm{~N}$ & $\mathrm{~N}$ & 0.55 & $\mathrm{LC}$ \\
\hline $\begin{array}{l}\text { Nasa cymbopetala (Urb. \& Gilg) } \\
\text { Weigend }\end{array}$ & 3 & 17 & 1 & 21 & A & $\mathrm{XX}$ & $\mathrm{B}$ & $\mathrm{N}$ & 1 & $\mathrm{~N}$ & $\mathrm{~N}$ & 0.37 & NT \\
\hline Nicotiana glutinosa $\mathrm{L}$. & 5 & 28 & 18 & 51 & A & $\mathrm{XX}$ & $\mathrm{B}$ & $\mathrm{N}$ & 2 & $\mathrm{~N}$ & $\mathrm{~N}$ & 0.41 & $\mathrm{LC}$ \\
\hline Nicotiana rustica L. & 3 & 18 & 46 & 67 & A & $\mathrm{XX}$ & $\mathrm{B}$ & $\mathrm{N}$ & 3 & $\mathrm{~N}$ & $\mathrm{~N}$ & 0.63 & $\mathrm{LC}$ \\
\hline Oenothera rosea L'Hér. ex Aiton & 11 & 22 & 282 & 315 & A & $\mathrm{XX}$ & M & $\mathrm{N}$ & 12 & $\mathrm{~N}$ & $\mathrm{~N}$ & 0.58 & $\mathrm{LC}$ \\
\hline $\begin{array}{l}\text { Otholobium pubescens (Poir.) J.W. } \\
\text { Grimes }\end{array}$ & 4 & 31 & 14 & 49 & A & $\mathrm{XX}$ & A & $\mathrm{N}$ & 1 & $\mathrm{~N}$ & $\mathrm{~N}$ & 0.40 & EN \\
\hline Perezia coerulescens Wedd. & 4 & 18 & 8 & 30 & A & $\mathrm{XX}$ & $\mathrm{A}$ & $\mathrm{N}$ & 2 & $\mathrm{~N}$ & $\mathrm{~N}$ & 0.37 & $\mathrm{CR}$ \\
\hline Perezia multiflora (Bonpl.) Less. & 6 & 25 & 54 & 85 & A & $\mathrm{XX}$ & A & $\mathrm{N}$ & 3 & $\mathrm{~N}$ & $\mathrm{~N}$ & 0.46 & $\mathrm{CR}$ \\
\hline Perezia pinnatifida (Bonpl.) Wedd. & 4 & 14 & 1 & 19 & A & $\mathrm{XX}$ & A & $\mathrm{N}$ & 1 & $\mathrm{~N}$ & $\mathrm{~N}$ & 0.43 & $\mathrm{VU}$ \\
\hline Plantago lanceolata $\mathrm{L}$. & 5 & 20 & 102 & 127 & B & $\mathrm{XX}$ & A & $\mathrm{N}$ & 9 & $\mathrm{~N}$ & $\mathrm{~N}$ & 0.43 & NT \\
\hline Plantago major $\mathrm{L}$. & 4 & 13 & 206 & 223 & A & $\mathrm{XX}$ & $\mathrm{A}$ & $\mathrm{N}$ & 14 & $\mathrm{~N}$ & $\mathrm{~N}$ & 0.33 & $\mathrm{LC}$ \\
\hline Plantago myosuros Lam. & 2 & 7 & 34 & 43 & A & $\mathrm{XX}$ & M & $\mathrm{N}$ & 4 & $\mathrm{~N}$ & $\mathrm{~N}$ & 0.36 & NT \\
\hline Polylepis racemosa Ruiz \& Pav. & 3 & 39 & 2 & 44 & A & $\mathrm{XX}$ & $\mathrm{B}$ & $\mathrm{N}$ & 1 & $\mathrm{~N}$ & $\mathrm{~N}$ & 0.51 & NT \\
\hline Ruellia floribunda Hook. & 7 & 23 & 17 & 47 & A & $\mathrm{XX}$ & $\mathrm{B}$ & $\mathrm{N}$ & 1 & $\mathrm{~N}$ & $\mathrm{~N}$ & 0.43 & NT \\
\hline Senecio cantensis Cabrera & 13 & 0 & 0 & 13 & A & $\mathrm{X}$ & $\mathrm{B}$ & $\mathrm{X}$ & 0 & $\mathrm{X}$ & $\mathrm{N}$ & 0.24 & $\mathrm{CR}$ \\
\hline Senecio collinus DC. & 11 & 6 & 0 & 17 & A & $\mathrm{X}$ & M & $\mathrm{X}$ & 0 & $\mathrm{X}$ & $\mathrm{N}$ & 0.28 & $\mathrm{CR}$ \\
\hline Senecio comosus Sch. Bip. & 2 & 23 & 6 & 31 & A & $\mathrm{XX}$ & M & $\mathrm{N}$ & 2 & $\mathrm{~N}$ & $\mathrm{~N}$ & 0.22 & $\mathrm{CR}$ \\
\hline Senecio nivalis (Kunth) Cuatrec. & 6 & 7 & 9 & 22 & A & $\mathrm{X}$ & A & $\mathrm{N}$ & 1 & $\mathrm{~N}$ & $\mathrm{~N}$ & 0.13 & $\mathrm{CR}$ \\
\hline Senecio rhizomatus Rugby & 1 & 10 & 0 & 11 & A & $\mathrm{X}$ & $\mathrm{A}$ & $\mathrm{X}$ & 0 & $\mathrm{~N}$ & $\mathrm{X}$ & 0.20 & $\mathrm{CR}$ \\
\hline Senecio richii A. Gray & 13 & 7 & 0 & 20 & A & $\mathrm{X}$ & $\mathrm{A}$ & $\mathrm{X}$ & 0 & $\mathrm{X}$ & $\mathrm{N}$ & 0.13 & $\mathrm{CR}$ \\
\hline Senecio yauyensis Cabrera & 8 & 13 & 0 & 21 & B & $\mathrm{X}$ & $\mathrm{B}$ & $\mathrm{X}$ & 0 & $\mathrm{~N}$ & $\mathrm{~N}$ & 0.11 & $\mathrm{CR}$ \\
\hline Sessea confertiflora Francey & 6 & 14 & sd & 20 & B & $\mathrm{XX}$ & M & sd & sd & sd & sd & 0.2 & DD \\
\hline Siphocampylus biserratus (Cav.) A. DC. & 11 & 17 & 1 & 29 & B & $\mathrm{XX}$ & $\mathrm{S}$ & $\mathrm{N}$ & 1 & $\mathrm{X}$ & $\mathrm{N}$ & 0.22 & VU \\
\hline Solanum americanum Mill. & 20 & 16 & 0 & 36 & A & $\mathrm{XX}$ & B & $\mathrm{X}$ & 0 & $\mathrm{~N}$ & $\mathrm{~N}$ & 0.65 & NT \\
\hline Solanum excisirhombeum Bitter & 4 & 13 & 2 & 19 & A & $\mathrm{XX}$ & M & $\mathrm{N}$ & 2 & $\mathrm{~N}$ & $\mathrm{~N}$ & 0.33 & NT \\
\hline Solanum nitidum Ruiz \& Pav. & 6 & 50 & 39 & 95 & A & $\mathrm{XX}$ & $\mathrm{B}$ & $\mathrm{N}$ & 2 & $\mathrm{~N}$ & $\mathrm{~N}$ & 0.62 & NT \\
\hline Solanum pentlandii Dunal & 10 & 17 & 4 & 31 & A & $\mathrm{XX}$ & $\mathrm{B}$ & $\mathrm{N}$ & 2 & $\mathrm{~N}$ & $\mathrm{~N}$ & 0.20 & NT \\
\hline Spartium junceum L. & 4 & 16 & 47 & 67 & A & $\mathrm{XX}$ & M & $\mathrm{N}$ & 5 & $\mathrm{~N}$ & $\mathrm{~N}$ & 0.40 & $\mathrm{LC}$ \\
\hline Spilanthes leiocarpa DC. & 1 & 6 & 1 & 8 & A & $\mathrm{XX}$ & M & $\mathrm{N}$ & 1 & $\mathrm{~N}$ & $\mathrm{X}$ & 0.26 & NT \\
\hline Tagetes elliptica Smith & 2 & 12 & 0 & 14 & A & $\mathrm{XX}$ & M & $\mathrm{X}$ & 0 & $\mathrm{X}$ & $\mathrm{N}$ & 0.13 & $\mathrm{CR}$ \\
\hline Tagetes filifolia Lag. & 2 & 17 & 111 & 130 & A & $\mathrm{XX}$ & M & $\mathrm{N}$ & 11 & $\mathrm{~N}$ & $\mathrm{~N}$ & 0.22 & $\mathrm{LC}$ \\
\hline Tagetes gracilis DC. & 3 & 7 & 0 & 10 & B & $\mathrm{XX}$ & $\mathrm{S}$ & $\mathrm{X}$ & 0 & $\mathrm{~N}$ & $\mathrm{~N}$ & 0.16 & EN \\
\hline $\begin{array}{l}\text { Tropaeolum tuberosum subsp. silvestre } \\
\text { Sparre }\end{array}$ & 14 & 11 & 79 & 104 & A & $\mathrm{XX}$ & $\mathrm{B}$ & $\mathrm{N}$ & 2 & $\mathrm{~N}$ & $\mathrm{~N}$ & 0.50 & NT \\
\hline Werneria caespitosa Wedd. & 2 & 12 & 1 & 15 & A & $\mathrm{XX}$ & M & $\mathrm{N}$ & 1 & $\mathrm{~N}$ & $\mathrm{~N}$ & 0.13 & $\mathrm{CR}$ \\
\hline $\begin{array}{l}\text { Xenophyllum decorum (S.F. Blake) } \\
\text { V.A. Funk }\end{array}$ & 2 & 7 & 0 & 9 & A & $\mathrm{X}$ & $\mathrm{S}$ & $X$ & 0 & $X$ & $\mathrm{~N}$ & 0.09 & $\mathrm{CR}$ \\
\hline
\end{tabular}

\footnotetext{
${ }^{1}$ Universidad Nacional Pedro Ruiz Gallo, Av. Juan XXIII 339, Lambayeque, Perú, horaciodlc@hotmail.com ${ }^{2}$ Universidad Nacional Agraria La Molina, apartado Postal 12-056 Lima 12, Perú, pazpolli@lamolina.edu.pe ${ }^{3}$ Universidad Nacional Agraria La Molina, apartado Postal 12-056 Lima 12, Perú, gvilcapoma@lamolina.edu.pe
} 